\title{
Antimicrobial properties of Chlorocardium rodiei on Pseudomonas aeruginosa, Bacillus spp. and Candida albicans
}

\author{
DENNIS DAVID", RUTH DANIEL ${ }^{\natural »}$, DIANA SEECHARRAN

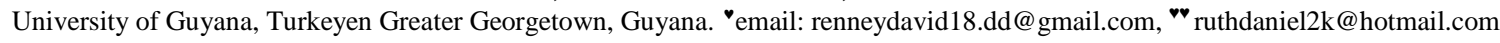

Manuscript received: 11 January 2019. Revision accepted: 19 June 2019.

\begin{abstract}
David D, Daniel R, Seecharran D, 2019. Antimicrobial properties of Chlorocardium rodiei on Pseudomonas aeruginosa, Bacillus spp. and Candida albicans. Nusantara Bioscience 11: 128-132. This research seeks to investigate the antimicrobial properties of Chlorocardium rodiei leaves and seeds on Pseudomonas aeruginosa, Bacillus spp. and Candida albicans. It also seeks to extract and identify the phytochemical compounds presented in leaves and seeds. The crude plant extract was obtained using the maceration method of extraction (ethanol and acetone as solvent) followed by rotary evaporation. Phytochemicals were analyzed from the crude extract using standard phytochemical procedures. $1 \%, 50 \%$ and $100 \%$ concentrations of each extract were prepared and used in the disk diffusion assay against $C$. albicans, P. aeruginosa, and Bacillus spp. and compared against Ciprofloxacin and Erythromycin for the bacteria and Fluconazole for the fungus. The results showed that the extract from the leaves possesses weak $(\leq 12$ mm) to moderate ( $>12$ $\leq 15 \mathrm{~mm})$ inhibitory effect, while the extract from the seeds had weak $(<12 \mathrm{~mm})$ inhibitory effect on the microorganisms. The phytochemical compounds tannins, essential oils, saponins, and phenols were tested positive from the crude extract. For the first time, the antimicrobial properties of $C$. rodiei's leaves and seeds were evaluated and showed to possessed antimicrobial properties.
\end{abstract}

Keywords: Antibiotics resistance, antimicrobial property, Chlorocardium rodiei, extraction

\section{INTRODUCTION}

Chlorocardium rodiei (Schomb.), formerly referred to as Ocotea rodiaei (Mez,) belongs to the plant family Lauraceae (Borges et al. 2008), which are aromatic evergreen trees and shrubs. This family is distributed globally but is commonly found in the tropics of America and Southeast Asia. Its habitat is the lowland forest but can also be found at higher altitude in tropical montane forest. It comprises of 30 genera with over 2000 species (Salleh et al. 2015). C. rodiei (Schomb) is found in the Guiana Shield and is known for its outstanding characteristics such as; extremely dense, durability in seawater and high commercial value. This tree is used globally for construction purposes such as; bridges, walkways, wharves, docks, and houses (Iwokrama Canopy Walkway, 2014). The stem is used as a febrifuge, to treat diarrhea, for the treatment of malaria and bitter tonic. The stem-bark contains the alkaloids berberine and nectandra, while the seed contains berberis acid (DeFilipps et al. 2004). Since its parts are used to treat various illnesses and possess these alkaloids, the antimicrobial property is being investigated in this study.

The world is heading towards an era where once susceptible bacteria that were controlled by antibiotics are now resistant, which is rising to threatening levels all over the globe. As antibiotics lose its power to control and kill bacteria, it is getting more difficult and even impossible to treat infections such as pneumonia, blood poisoning, tuberculosis, gonorrhea and foodborne diseases (World Health Organization, 2017). The random use of antibiotics had allowed the bacterial resistant community to increase which result in infection taking a longer time to heal due to drug therapy losing its effectiveness (Volpato et al. 2017). Resistance of bacteria can be transmitted through the consumption of meat not properly cooked, exposure to the animals' manure, and contact with uncooked meat or surfaces meat has touched. This is due to these animals are treated with medically important antibiotics for growth improvement and infection prevention (Martin et al. 2015).

Thus, the ability of modern medicine to treat common infections is at risk, so there is a possibility that the $21^{\text {st }}$ century standard treatment will no longer be effective and there will be an increase in death from infections. We are able to live longer and healthier because of antibiotics. The calls for immediate and significant actions have to be taken in the application of production, prescription, and consumption of antibiotics to help us continue living longer and healthier. The resistance to antibiotics to the healthcare-communities is not new, e.g. $90 \%$ of Staphylococcus aureus in the United States is reported to be resistant to methicillin (often abbreviated MRSA) and in 36 countries the last resort for gonorrhea is proving less effective (Bernstein 2014). Pseudomonas aeruginosa is frequently associated with infections of the urinary and respiratory tracts in humans and also causes infection that is common in patients receiving treatment for severe burns. As a pathogen, it appears to be primarily an opportunist, initiating infections in individuals whose resistance is low. This organism is naturally resistant to many of the widely used antibiotics, so chemotherapy is often difficult. Resistance is frequently due to a resistance factor ( $R$ factor), which is a carrying genes coding for detoxification of various antibiotics (Brock et al. 1984). Pathogenic bacterial infections such as $P$. aeruginosa result in the 
death of a significant number of children and elderly. Although antimicrobial therapy has increased there is still significant increase in the rate of diseases causing death. With increasing resistance of bacteria to available antibiotics, the situation is even more elevated. Bacteria that are resistant to several antibiotics threatened the health of humans and other animals (Iqbal et al. 2013). The antimicrobial property has been carried out from many plants. Bhalodia and Shukla (2011) investigated secondary metabolites from plants with antimicrobial properties such as tannins, terpenoids, alkaloids, flavonoids, and glycosides. Plants produce bioactive molecules that allow them to interact with other organisms in the environment (Truiti et al. 2006)

World health leaders recognized that humans all over the world face global health threats from microorganisms that are resistant to antimicrobial drugs, such as Methicillin-Resistant Staphylococcus aureus (MRSA) and the multidrug- resistant $P$. aeruginosa (Center for Disease Control and Prevention 2013). The need for new drugs are of imminent importance and natural products derived from medicinal plants are promising sources, either as pure compounds or standardized extract (Kahaliw et al. 2017). Plant contains large variety of chemical compounds, as a result, they are sources of possible new drug leads (Kahaliw et al. 2017). In addition, they are the richest bioresource of drugs of traditional medicine, modern medicine, nutraceuticals, food supplements, folk medicines, drug intermediates, and starting point for synthetic drugs (Ncube et al. 2008).

The array of compounds with unique structures which plants produce has served as a stimulus for continued search for numerous antibiotics (Nickell 1959). Alseodaphne andersonii, a Laurel plant leaves were investigated for antimicrobial activity by Pracha et al. (2007), found it possessed antimicrobial activities. Therefore an attempt is being made in this research to find possible new leads of antibiotic and antifungal agents from Chlorocardium rodiei (Schomb.) (Greenheart) leaves and seeds.

\section{MATERIALS AND METHODS}

\section{Site of collection and identification of plant}

Plant materials were collected during the period of December 2017 - January 2018 from Kkaburi village, 72 Miles Bartica Potaro Road, Region \#7, Guyana. Samples were taken to the Center for Study of Biological Diversity (CSBD) at the University of Guyana for verification.

\section{Preparation and extraction of plant materials}

Leaves and seeds were washed with water to remove any foreign particles attached to the plant materials. Plant materials were then dried in oven at $45^{\circ} \mathrm{C}$ until constant weights were achieved. The dry materials were ground into powder, weighed and placed in a clean container for further use. Extraction using maceration method with two different solvents (acetone and ethanol) was carried out according to the method by Tiwari et al. (2011). The extract was then concentrated using a rotary evaporator to obtain crude extract.

\section{Preparation and storage of culture media (Mueller Hinton Agar).}

The culture media was prepared according to manufacturer's instruction under aseptic conditions and Tendencia, (2004) method of storage was followed with some modification. Petri plates were sterilized under laminar air-flow cabinet using UV light. Plates were poured on a level surface to give a uniform depth of $4 \pm 0.5 \mathrm{~mm}$ under the laminar air flow cabinet and an aseptic environment was maintained. The plates were then left to solidify, placed in airtight bags and stored at $4^{\circ} \mathrm{C}$ in a refrigerator in an inverted position. Prior to inoculation, plates were dried in hot air oven at $120^{\circ} \mathrm{F}$ and cooled to room temperature for approximately 1 hour.

\section{Source and preparation of microbial culture \\ Bacterial and fungal cultures}

Isolates of bacteria (P. aeruginosa and Bacillus sp.) and fungi (Candida albicans) were obtained from Eureka Medical Laboratory, Georgetown. The microbial cultures were subcultured unto Mueller Hinton Agar (MHA) medium using the streak method to obtain isolated colonies. The sub-cultures were then incubated at $37^{\circ} \mathrm{C}$ for 24 hours for the bacteria and 48 hours for fungi then refrigerated at $4^{0} \mathrm{C}$ until further use. Inoculums of bacteria and fungi were prepared by suspending colonies in sterile distilled water and mixed to even turbidity and compared to $0.5 \mathrm{Mc}$ Farland Standard according to the method by Tendencia (2004) with some modifications.

\section{Disk diffusion assay}

Tendencia (2004) method of inoculation of plates and application of disks was also followed. Whatman \#1 filter paper $(6 \mathrm{~mm})$ was used and impregnated with plant extracts at three different concentrations, $1 \%, 50 \%$, and $100 \%$ respectively a day before application on growth media. After applying overnight impregnated discs, plates were then incubated at $37^{\circ} \mathrm{C}$ for 24 hours for bacteria and 48 hours for fungus. Antimicrobial disks, fluconazole, erythromycin, and ciprofloxacin were used as references. Reading of plates followed the European Committee on Antimicrobial Susceptibility Testing (EUCAST) Reading Guide Version 5 (EUCAST 2017).

\section{Phytochemical analysis}

Phytochemical analysis of ethanol and acetone extract of leaves and seeds of $C$. rodie $i$ was carried out according to the standard phytochemical analysis procedures as follows: phenols and alkaloids (Tiwari et al. 2011), steroids (Pandey et al. 2014), tannins and saponins (Hossain 2013) and volatile oils (Deshpande et al. 2014).

\section{Data analysis}

Using R-studio, version 3.5.2, a one-way ANOVA test was used to analyze the data with a level of significance set at $\alpha=0.05$. The data was recorded as mean \pm standard deviation. 


\section{RESULTS AND DISCUSSION}

The results of this research are presented in Table 1-2. The leaves extracts were statistically different $(p<0.05)$ from seeds extracts in inhibiting the growth of the three microorganisms. There was also a statistical difference $(\mathrm{P}<$ 0.05) between the solvents ethanol and acetone in inhibiting the growth of the microorganism. In addition, the reference treatment was statistically different from plant extracts $(\mathrm{p}<0.05)$. C. albicans was significantly susceptible to the leaves extract than $P$. aeruginosa and Bacillus sp. but not statistically different. There was no statistical difference amongst microorganism's susceptibility to the extracts of the seeds (Figure 1).

In this study, $C$. rodiei leaves and seeds were tested for six antimicrobial classes of phytochemical compounds (steroids, alkaloids, saponins, volatile oils, phenols, and tannins) and the antimicrobial activities evaluated using the disk diffusion method. However, only three of these compounds were present in the acetone leaves extract in addition to ethanol and acetone seed extracts namely; saponins, volatile oils and phenols (Table 2). These compounds have different mechanism of action as antimicrobial. Phenols and tannins bind to adhesins, enzyme inhibition, substrate deprivation, form complex with cell wall, membrane disruption and form metal ion complexation. Volatile oil, on the other hand, acts on cell membrane disruption only (Tiwari et al. 2011) while saponins permeabilize cell membrane (Jacob et al. 1991).

When the average zone of inhibition from the seeds and leaves were compared, the leaves extract showed to have more effects on the microorganisms being tested. This effect can be due to the number of compounds that are extracted with ethanol as compared to acetone, (Table 2). According to Tiwari et al. (2011), ethanol extracts the following active compounds namely; tannins, polyphenols, flavonol, alkaloids, sterols, terpenoids, and polyacetylenes, while acetone extracts phenols, flavonols and saponins. Although both solvents are polar in nature they vary in degree of polarity and differ in the number and kind of compounds they can extract. Alcohols, under which ethanol falls, are effective in cell wall degradation and caused polyphenols to be released from cells and is easier to penetrate the cellular membrane to extract the intercellular ingredients from the plant material (Ijaiya et al. 2014). This explanation corroborates with the results obtained by Kotze et al. (2002), highlighting ethanol extract having more inhibitory effects than acetone and proved to be more effective in extracting phytochemical.

Table 2. Phytochemicals found present in the different plant extracts

\begin{tabular}{|c|c|c|c|c|}
\hline & \multicolumn{2}{|c|}{ Leaves extracts } & \multicolumn{2}{|c|}{ Seeds extracts } \\
\hline Phytochemicals & Ethanol & Acetone & Ethanol & Acetone \\
\hline Alkaloids & - & - & - & - \\
\hline Tannins & + & - & - & \\
\hline Saponins & + & + & + & + \\
\hline Volatile oils & + & + & + & + \\
\hline Phenols & + & + & + & + \\
\hline Steroids & - & - & - & - \\
\hline
\end{tabular}

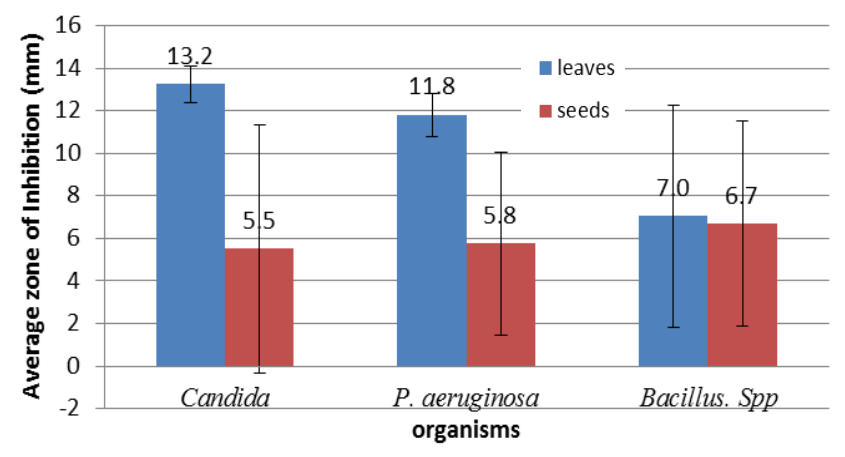

Figure 1. Differences in zone of inhibition from leaves and seed extracts on the various organisms

Table 1. Zone of inhibition of ethanol and acetone leaves and seeds extract of C.rodiei

\begin{tabular}{|c|c|c|c|c|c|c|c|c|c|c|}
\hline \multirow{3}{*}{ Organism } & \multirow{3}{*}{ Solvent } & \multicolumn{9}{|c|}{ Zone of inhibition (Mean \pm Standard Deviation) $\mathbf{m m}$} \\
\hline & & \multicolumn{2}{|c|}{$1 \%$} & \multicolumn{2}{|c|}{$\mathbf{5 0 \%}$} & \multicolumn{2}{|c|}{$100 \%$} & \multicolumn{3}{|c|}{ References } \\
\hline & & Seeds & Leaves & Seeds & Leaves & Seeds & Leaves & Fluconazole & Erythromycin & Ciprofloxacin \\
\hline \multirow{2}{*}{ C. albicans } & Ethanol & $13.7 \pm 6.2$ & $12.7 \pm 2.5$ & $11.9 \pm 1.5$ & $13 \pm 1.6$ & $7.4 \pm 1.8$ & $14.7 \pm 2.9$ & $29.3 \pm 4.5$ & & \\
\hline & Acetone & 0 & $11.8 \pm 0.4$ & 0 & $13.8 \pm 1.9$ & 0 & $13.5 \pm 1.9$ & & & \\
\hline \multirow[t]{2}{*}{$P$. aeruginosa } & Ethanol & $15.9 \pm 2.1$ & $12.8 \pm 2.1$ & $8.9 \pm 0.4$ & $11.7 \pm 1$ & $6.3 \pm 0.9$ & $12.8 \pm 0.7$ & & $27.3 \pm 0.6$ & $33.7 \pm 2.1$ \\
\hline & Acetone & 0 & $11.9 \pm 0.6$ & $5.7 \pm 1.1$ & $11.8 \pm 1.2$ & $1.2 \pm 1.6$ & $9.8 \pm 1.6$ & & & \\
\hline \multirow[t]{2}{*}{ Bacillus sp. } & Ethanol & 0 & 0 & $12 \pm 0.6$ & $7.6 \pm 1.2$ & $9.7 \pm 0.8$ & $10.7 \pm 0.3$ & & $38.5 \pm 0.5$ & $32.7 \pm 0.6$ \\
\hline & Acetone & 0 & 0 & $9.3 \pm 0.3$ & 0 & $9.1 \pm 1.0$ & $12.8 \pm 2.6$ & & & \\
\hline
\end{tabular}

Note: Results are expressed as mean \pm standard deviation from three trials. The diameter of the disk $(\emptyset=6 \mathrm{~mm})$ was included 
However, Alothyqi et al. (2016) showed that acetone is more effective in extracting phytochemicals compounds since it has an intermediate polarity and has been reported that such solvents are more efficient in extraction of bioactive compounds than polar and non- polar solvents. On the contrary, there are a number of factors that affect the extraction process of phytochemical compounds using solvents. These factors include; genetic constitution, age of plant, parts of plant and physiological adaptation of plants to their various environment and based on these factors the efficiency of the solvent varies and can prevent phytochemical compounds to be extracted. Ijaiya et al. (2014) also noted that variations of phytochemical compounds that can be extracted depend on solvent used for extraction.

Due to the simple structural makeup of fungus cell wall which compromises primarily of chitin, C. albicans is more susceptible to the leaves extracts than $P$. aeruginosa and Bacillus sp. which have more complex cell wall structure. Its susceptibility is also supported by the fact that the presence of tannins found in the ethanol extracts and not in the others, gives us a possible explanation of why $C$. albicans is more susceptible to it than the other microorganisms. However, Bacillus sp. is more susceptible to the seed extracts than $P$. aeruginosa while $C$. albicans was only sensitive to ethanol seed extract. This is probably due to the differences in outer membrane surrounding the cell wall in gram-negative bacteria that prevents diffusion of antimicrobial compounds through its lipopolysaccharide covering. In addition, gram-negative bacteria also produce enzymes that are capable of digesting foreign compounds introduced inside their cell (Klancnik et al. 2010).

With an increase in concentration, there is an increase in zone of inhibition for $C$. albicans primarily because of its simple cell wall and possibly the presence of more active compound as the concentration increases. Al-Manhel $\&$ Niamah (2015) found a similar trend with the 5 species of fungi that were investigated. Klancnik et al. (2010) noted that the absence of zone of inhibition around a disk using the disk diffusion method can be because of the presence of less polar compounds that diffuses more slowly in the culture medium and the hydrophobic nature of some compounds. In such cases, other methods may be used.

According to the range utilized by Marotta et al. (2016) to determine antimicrobial activity, this study showed that the leaves of $C$. rodiei had weak (inhibition zone $\leq 12 \mathrm{~mm}$ ) to moderate $(12 \quad \mathrm{~mm}<$ inhibition zone $<20 \quad \mathrm{~mm})$ antimicrobial activities, while the seeds only showed weak activity (inhibition zone $\leq 12 \mathrm{~mm}$ ) and none of the extracts showed strong activity (inhibition zone $\leq 20 \mathrm{~mm}$ ) on all microorganisms tested except the reference treatments. These activities are due to the phytochemicals found present in the parts of the plant. As compared to the phytochemicals found, other researchers noted the presence of similar compounds that showed antimicrobial properties in the Lauraceae plant family. Kahaliw et al. (2017) highlighted the main chemical constituents found in the leaves of Persea americana as volatile oils, flavonoids, and coumarins. In addition, Pandey et al. (2014), evaluated the phytoconstituents of Cinnamon zeylanicum bark extract and found the following phytochemicals namely; steroids, alkaloids, flavonoids, saponins, tannins, and phenols. Pracha et al (2007) found Alseodaphne andersonii leaves to contain alkaloids, glycosides, steroids, protein and amino acids. Prabuseenivasan et al. (2006), showed that Cinnamomum camphora and Cinnamomum zeylanicum are active against $P$. aeruginosa $(33.3 \mathrm{~mm})$, and $B$. subtilis (29.9 mm). Dabas et al. 2013; Evelin et al. 2016, also showed that Persea americana seeds have antimicrobial property. This study showed that $C$. rodiei seeds also possessed antimicrobial activities.

However, because there is difference in methodologies used in different studies, it becomes difficult to compare results for antimicrobial plant extracts. The accuracy to compare results is difficult since each method is based on its own principles. The results obtained are influenced by a number of factors including choice of method, microorganism used, extraction technique and the degree of solubility of each test compounds (Klancnik et al. 2010).

\section{REFERENCES}

Al-Manhel AJ, Niamah AK. 2015. Effect of aqueous and alcoholic plant extracts on inhibition of some types of microbes and causing spoilage of food. J Nutr Food Sci 5 (006). DOI:10.4172/2155-9600.S5-00.

Alothyqi N, Almalki M, Albqalai M, Alsamiri H, Alrashdi SM, et al. 2016. In vitro antibacterial activity of four Saudi medicinal plants. J Microb Biochem Technol 8 (2): 083-089. DOI:10.4172/19485948.100026

Bernstein L. 2014. Increasing antibiotic resistance could have disastrous effects, WHO warn. Washington Post. http://bangordailynews.com/2014/05/01/health/increasing-antibioticresistance-could-have-disastrous-effects-health-organization-warns/

Bhalodia NR, Shukla VJ. 2011. Antibacterial and antifungal activities. Pharm Technol Res 2 (2): 104-109. DOI:10.4103/2231-4040.82956.

Borges LMS, Cragg SM, Bergot J, Williams JR, Shayler B, Sawyer GS. 2008. Laboratory Screening of tropical hardwoods for natural resistance to the marine borer Limnoria quadripunctata: The role of leachable and non-leachable factors. Holzforschung 62: 99-111. DOI:10.1515/HF.2008.015.

Brock TD, Smith DS, Madigan MT. 1984. Biology of Microorganisms ( $4^{\text {th }}$ ed.). Prentice-Hall, Inc. New Jersey.

Center for Disease Control and Prevention. 2013. Antibiotics resistance threats in the United States, 2013. https://www.cdc.gov/drugresistance/threat-report-2013/index.html.

Dabas D, Shegog RM, Ziegler GR, Lambert JD. 2013. Avocado (Persea americana) Seed as a source of bioactive phytochemicals. Curr Pharm Des 19: 6133-6140. DOI:10.2174/1381612811319340007.

DeFilipps RA, Maina SL, Crepin J. 2004. Medicinal plants of the Guianas. http://botany.si.edu/bdg/medicinal/

Deshpande PK, Gothalwal R, Pathak AK. 2014. Phytochemical analysis and evaluation of antimalarial activity of Azadirachta indica. Pharm Innov J 3 (9): 12-16.

European Committee on Antimicrobial Susceptibility Testing. 2017. Reading Guide. http://www.eucast.org/ast_of_bacteria/disk_diffusion_methodology/

Evelin Y, Luis M, Ana M, Luz M. 2016. Evaluation of the antimicrobial capacity of avocado seed (Persea americana. Mill): Potential application of by-products. Vitae 23: 143-144.

Hossain MA, Al-Raqmi KAS, Al-Mijizy ZH, Weil M, Al-Riyami Q. 2013. Study of total phenol, flavonoids contents and phytochemical screening of various leaves crude extracts of locally grown Thymus vulgaris. Asian Pacific J Trop Biomed 3 (9): 705-710.

Ijaiya IS, Arzika S, Abdulkadir M. 2014. Extraction and Phytochemical screening of the roots and leaves of Annona senegalesis (Wild custard apple). Academic J Interdiscip Stud 3 (7). DOI:10.5901/ajis.2014.v3n7p9. 
Iqbal J, Siddiqui R, Kazami SU, Khan NA. 2013. A Simple assay to screen antimicrobial compounds potentiating the activity of current antibiotics. BioMed Res Int. DOI: 10.1155/2013/927323

Iwokrama Canopy Walkway. 2014. Flora and Fauna of Iwokrama https://iwokramacanopywalkway.com/wp-

content/uploads/2014/03/Iwokrama-Canopy-Walkway-SpeciesList.pdf?lightbox $[$ width $]=600 \&$ lightbox [height $]=800 \&$ lightbox $[$ ifram e]=true.

Jacob MC, Faver M, Bensa JC. 1991. Membrane cell permeabilization with saponin and multiparametric analysis by flow cytometry. Cytometry 12 (6): 550-558.

Kahaliw W, Aseffa A, Abebe M, Teferi M, Engidawork E. 2017 Evaluation of the antimycobacterial activity of crude extracts and solvents fractions of selected Ethiopian medicinal plants. BMC Complement Altern Med 17 (143). DOI 10.1186/s12906-017-1563-0.

Klancnik A, Piskernik S, Jersek B, Mozina SS. 2010. Evaluation of diffusion and dilution methods to determine the antibacterial of plan extract. J Microb Methods 81: 121-126.

Kotze M, Eloff JN, Houghton PJ. 2002. Extraction of antibacterial compounds from Combretum microphyllum (Combretaceae). South Afr J Bot 66 (1): 62-67.

Marotta SM, Giarratana F, Parco A, Neri D, Ziino G, Giuffrida A, and Panebinaco A. (2016) Evaluation of the Antibacterial Activity of Bergamot Essential Oils on Different Listeria Monocytogenes Strains. Italian J Food Sci 5(4): 6176, doi: 10.4081/ijfs.2016.6176

Martin MJ, Thottathil SE, Newman TB. 2015. Antibiotics overuse in animal agriculture: A call for action for health care provider. Am J Publ Health 105 (12): 2409-2410.

Ncube NS, Afolayan AJ, Okoh AI. 2008. Assessment techniques of antimicrobial properties of natural compounds of plant origin: current methods and future trends. Afr J Biotechnol 7 (12): 1797-1806.
Nickell LG. 1959. Antimicrobial activity of vascular plants. Econ Bot 13 (4): 281-318.

Pandey S, Pandey R, Singh R. 2014. Phytochemical screening of selected medicinal plant Cinnamon zeylanicum bark extract, area of research; Uttarakhand, India. Int J Sci Res Publ 4 (6). DOI: 10.1080/13880200601028313.

Prabuseenivasan S, Jayakumar M, Ignaciuthu S. 2006. In Vitro antibacterial activity of some plant essential oils. BMC Compl Altern Med. DOI:10.1186/1472-6882-6-39.

Pracha V, Kaushik A, Rawat MSM, Biswas SAS. 2007. Evaluation of antimicrobial potential of Alesodaphne andersonii leaf extracts against pathogenic bacteria. Pharm Biol 45 (1): 60-63.

Salleh WMNHW, Ahmad F, Yen KH, Zulkifli RM. 2015. Chemical composition and biological activities of essential oil of Beilschmiedia pulverulenta. Pharm Biol 54 (2): 322-330.

Tendencia EA. 2004. Chapter 2. Disk Diffusion Method. http://hdl.handle.net/10862/1635

Tiwari P, Kumar B, Kaur M, Kaur G, Kaur H. 2011. Phytochemical screening and extraction: A review. Internationale Pharm Sci 1 (1). http://ipharmsciencia.com

Truiti MT, Bersani-Amado CA, Filho BPD, Sarragiotto MH, Conceicao de Souza M. 2006. Screening of five Brazilian plants for antiinflammatory and antimicrobial activities. Pharm Biol 44 (7): 516521.

Volpato L, Gabardo MCL, Leonardi DP, Tomazinho PH, Maranho LT, Baratto-Filho F. 2017. Effectiveness of Persea major Kopp (Lauraceae) extract against Enterococcus faecalis: a preliminary in vitro study. BioMed Central 10 (119). DOI:10.1186/s13104-0172443-x.

World Health Organization. 2017. Antibiotic Resistance. http://www.who.int/mediacentre/factsheets/antibiotic-resistance/en/ 